\title{
Rheological Properties of Hyaluronic Acid Diluted Solutions as Components of Cosmetics
}

\author{
Svetlana Saitarly ${ }^{(\mathbb{D})}$, Yuriy Pushkarev ${ }^{1 \mathbb{D}}$, Mariia Nesterkina ${ }^{1, * \mathbb{D}}$, Serhat Öztürk $^{2 \mathbb{C}}$, Bekir Salih ${ }^{2 \mathbb{D}}$, \\ Iryna Kravchenko 1 (iD \\ 1 Department of Organic and Pharmaceutical Technology, Odesa National Polytechnic University, Odesa 65044, Ukraine \\ 2 Department of Chemistry, Hacettepe University, Ankara 06800, Turkey \\ * Correspondence: mashaneutron@gmail.com (M.N.);
}

Scopus Author ID 56197250700

Received: 14.03.2021; Revised: 3.05.2021; Accepted: 8.05.2021; Published: 10.06.2021

\begin{abstract}
Rheological measurements were carried out on dilute aqueous solutions of high (HMW-HA, $1150 \mathrm{kDa}$ ) and low molecular weight hyaluronic acids (LMW-HA, $8 \mathrm{kDa}$ ) along with their mixtures to determine the relationship between the viscosity and concentration of the samples. Molecular weight and polydispersity of low molecular HA have been determined by matrix-assisted laser desorption ionization mass spectrometry (MALDI-MS). HA diluted solutions were found to be weakly structured liquids with a slight viscosity anomaly. Critical values of concentration were determined at different ratios of HMW-HA and LMW-HA, leading to a sharp increase in the viscosity of solutions. Rheological studies have proved the pseudoplastic nature of the viscous flow of HMW-HA solutions. The possibility of using the Ostwald-de Waele equation for the mathematical description of a viscous flow was established. The results of the rheological analysis might be applied for the rational development of cosmetic formulations containing hyaluronic acid of various molecular weights.
\end{abstract}

Keywords: hyaluronic acid; molecular weight; MALDI; viscosity; rheological properties; technology.

(C) 2021 by the authors. This article is an open-access article distributed under the terms and conditions of the Creative Commons Attribution (CC BY) license (https://creativecommons.org/licenses/by/4.0/).

\section{Introduction}

Hyaluronic acid (HA) is one of the most popular high molecular weight compounds used in medicine and cosmetology. The range of HA applications is widespread and constantly being replenished, leading to increased demand for this biopolymer. HA possesses antimicrobial and regenerative effects, performs the function of a structural element, provides lubrication of joint moving parts, reducing their wear, plays an important role in embryogenesis, a transmitter of cellular mobility signals, etc. [1-3]. As the properties of HA are studied, new areas and prospects for its use are explored.

A number of researches are devoted to the HA action on connective tissues [4], its role in aging, restoration and rejuvenation of the skin [5,6], the application as injection implants including contour plastics of soft tissues, the prospects of cross-linked hydrogels usage as a basis for bone-plastic materials [7,8]. Additionally, physicochemical and pharmacological properties of HA were studied, the mechanisms of implementation, therapeutic action in dermatology, cosmetology, ophthalmology, orthopedics and other fields of medicine are described [9-11]. HA solutions were found to exhibit special rheological properties allowing the polymer to be a viscoelastic gel. HA molecular weight depends on the production source, varies within a wide range from $5 \mathrm{kDa}$ to $2 \mathrm{MDa}$ and determines its biological effect. Low https://biointerfaceresearch.com/ 
molecular weight HA (LMW-HA) penetrates well into the skin, affects the gene-regulating function of proteins, while high molecular weight HA (HMW-HA) prevents dryness [12]. Along with sulphoxides, alcohols and alkanols, terpenes and pyrrolidones, HA facilitates topical drug absorption [13-16].

The main structural unit of HA is a monomeric fragment - a disaccharide with a molecular weight of $400 \ldots 450 \mathrm{Da}$. Therefore, despite the different sources of origin due to the absence of isomerism, the chemical composition of HA polysaccharide is always constant and the polymer differs only in its molecular weight [17]. Oligosaccharides containing 10 monomeric disaccharide units possess an anti-inflammatory effect and those with 150-500 units - activate intracellular signaling processes $[18,19]$. In turn, molecules containing 1500 monomeric units form solutions with high viscosity and, consequently, take part in constructing the dermis extracellular matrix, particularly in the collagen network's structural organization [20]. An increase of macromolecule size to 2000 monomeric units leads to the growing role of HA in maintaining the dermis hydrobalance (optimal moisture level) and medium viscosity. At the same time, LMW-HA exhibits maximum antioxidant activity [21].

The polyanionic HA molecules are highly hygroscopic and represent the basic structure for organizing the extracellular medium's gel-like component; HA also participates in transportation and distribution of water molecules in body tissues [22,23]. For the most effective and safe application of HA preparations as intra-articular injections, essential are their certain physical and mechanical characteristics, particularly indicators of dynamic viscosity and viscoelastic properties. Bearing in mind the aforementioned, the aim of the present study was rheological measurements of compositions based on high (HMW-HA) and low (LMWHA) molecular weight hyaluronic acids as the main components of cosmetics.

\section{Materials and Methods}

High (molecular weight $1150 \mathrm{kDa}$ ) and low molecular HA were obtained from Truthful Concepts and Nutritional Integrity (Texas, USA). Molecular weight and polydispersity of low molecular HA were determined by matrix-assisted laser desorption ionization mass spectrometry (MALDI-MS). MALDI-MS analyses were performed on Bruker Rapiflex MALDI-TOF/TOF mass spectrometer (Bruker Daltonics, Bremen, Germany) equipped with a Smartbeam 3D ND:YAG $(355 \mathrm{~nm})$ laser in reflector-positive ion mode. The MALDI matrix, 2,5-dihydroxybenzoic acid (DHB), was dissolved in a 1:1 mixture of ACN and water containing $0.1 \%$ TFA (v/v) and used as the matrix. Sample solutions $(0.5 \mathrm{mg} / \mathrm{mL}$ dissolved in water) were mixed with DHB solution $(10.0 \mathrm{mg} / \mathrm{mL})$ in a ratio of $1: 5(\mathrm{v} / \mathrm{v})$. The sample/matrix mixtures $(0.5 \mu \mathrm{L})$ were deposited onto the MALDI sample target and allowed to dry at ambient conditions. The mass calibration on the instrument was performed with Peptide Calibration Standard II (Bruker Daltonics). FlexControl 4.0 and FlexAnalysis 4.0 (Bruker Daltonics) software were used to MS acquisition and process of MALDI-TOF data. MALDI matrix, 2,5Dihydroxybenzoic acid (DHB), acetonitrile (ACN), trifluoroacetic acid (TFA) were purchased from Sigma-Aldrich (St. Louis, MO, USA).

To study the effect of the relative molecular mass on viscous-flow properties, the mixtures of HMW-HA and LMW-HA aqueous solutions were prepared at their ratio from $20 / 80$ to $80 / 20$. The following diluted solutions with the concentration of $0.125 \% ; 0.0625 \%$; $0.0312 \%$ and $0.0156 \%$ were used for relative viscosity determination by Ubbelohde viscometer at $20 \pm 0.5^{\circ} \mathrm{C}$. Shear stress $\tau$ was determined on a rotary viscosimeter RHEOTEST-2 equipped with a thermostatic chamber in the temperature range $20-35{ }^{\circ} \mathrm{C}$ and calculated by the formula: 


$$
\tau=0.1 \cdot z \cdot \alpha
$$

where $\mathrm{z}$ - cylinder constant given in the instrument passport; $\alpha$-indication of device scale; 0.1 - shear stress conversion factor to Pa.

The dynamic viscosity of solutions $(\mathrm{Pa} \cdot \mathrm{s})$ was calculated from the ratio $\eta=\tau / \dot{\gamma}$, where $\tau$ - shear stress $(\mathrm{Pa}) ; \dot{\gamma}-$ shear rate gradient $\left(\mathrm{s}^{-1}\right)$.

\section{Results and Discussion}

For the characterization of low and high molecular weight HA, MALDI-MS was used to examine the molecular weight, monomer units and the polydispersity of HA. For this purpose, 2,5-dihydroxybenzoic acid MALDI matrix was found to be the best matrix for the characterization of HA among many different MALDI matrices tried in this study. For the high molecular weight HA, no resolved and reasonable signals were observed. It is impossible to observe high molecular weight polysaccharides in MALDI-MS even if in linear mode. For this reason, a reasonable MALD-MS signal for high molecular weight HA could not be obtained. In the case of low molecular weight HA, nice MALDI-MS signals were obtained in reflectron mode with high resolution. The MALDI-MS spectrum was acquired for low molecular weight HA, and the MALDI-MS spectrum is given in Figure 1. It can be seen from Figure 1 that the repeating unit of low molecular weight HA has 401 Da mass and containing two glycans, as seen in Scheme 1. From Scheme 1, it concluded that the main repeating unit mass was $401 \mathrm{Da}$, but it also contains two subunits with 203 and $198 \mathrm{Da}$, respectively. All results showed that low molecular weight HA had a main repeating unit with 401 Da mass but two subunits with 203 and 198 Da mass, respectively. The same appearance was observed from the MALDI-MS spectrum is given in Figure 1. Using Polytools software available on our Bruker Rapiflex MALDI-TOF/TOF mass spectrometer (Bruker Daltonics, Bremen, Germany), we calculated the molecular weight $\left(\overline{M_{n}}=3.2 \times 10^{3}\right)$ and the polydispersity was found to be 1.2 .

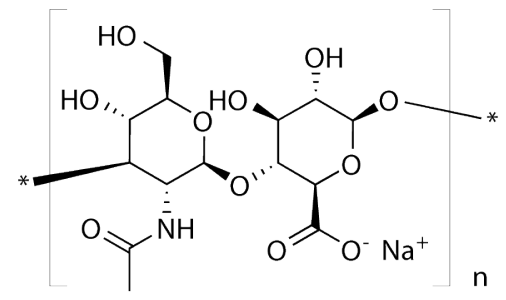

Scheme 1. Structural formulae of repeating unit of HA. Mass of the first unit $\left(\mathrm{C}_{8} \mathrm{H}_{13} \mathrm{NO}_{5}\right)$ is $203 \mathrm{Da}$ and the second unit $\left(\mathrm{C}_{6} \mathrm{H}_{7} \mathrm{O}_{6} \mathrm{Na}\right)$ is 198 totally 401 .

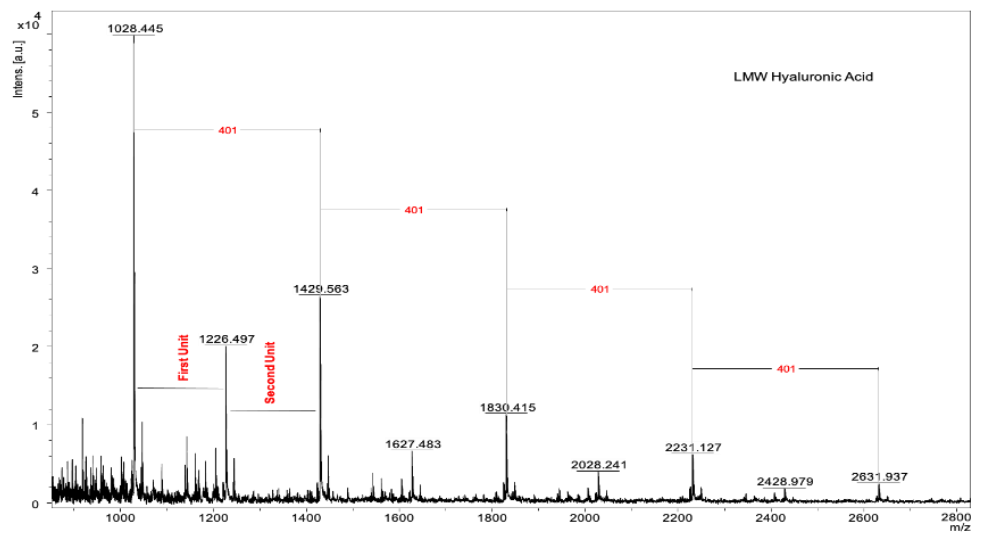

Figure 1. Positive ion and reflectron mode MALDI mass spectrum of low molecular weight hyaluronic acid. 
The viscosity of highly LMW-HA diluted solutions (0.0156-0.125\%) slightly (1.2-2.0 times) exceeds the viscosity of a pure solvent - distilled water. The short length of LMW-HA molecular chains $\left(\overline{M_{n}}=10^{3}\right)$ at low concentrations ensures that macromolecules are in solution practically isolated from each other. The addition of HMW-HA with significantly longer macromolecule chains $\left(\overline{M_{n}}=10^{6}\right)$ to LMW-HA solution increases the viscosity, which also grows with the concentration rising (Figure 2).

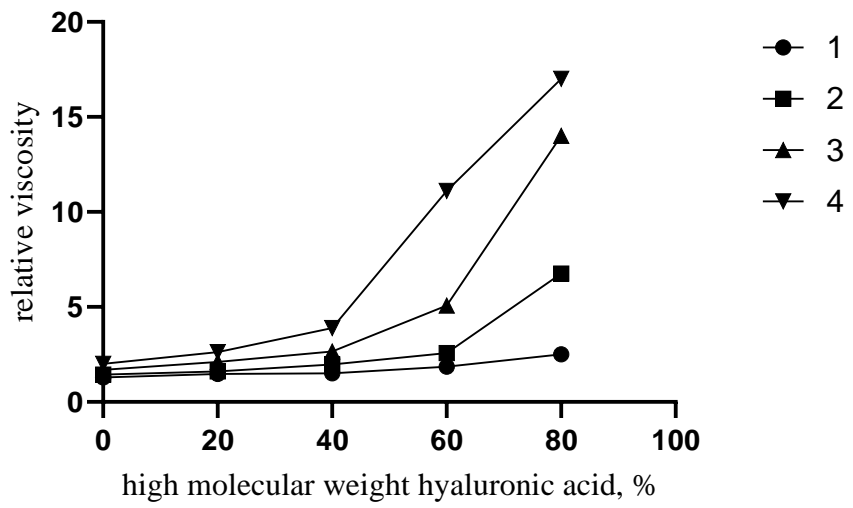

Figure 2. Relationship between the relative viscosity of LMW-HA/HMW-HA aqueous solutions and the HMWHA content. Solution concentrations: $1-0.0156 \% ; 2-0.0312 \% ; 3-0.0625 \% ; 4-0.125 \%$.

At a certain (critical) content of HMW-HA, the viscosity of solutions with a concentration of $0.0312-0.125 \%$ increases sharply. The critical value of HMW-HA content decreases with the concentration rising. At concentrations of $0.0312 \%$ and $0.0625 \%$, it manifests itself at 60\% and 50\% HMW-HA content (lines 2 and 3) and at a concentration of $0.125 \%$ - at $40 \%$ acid content (line 4 ). In a highly diluted solution with a concentration of $0.0156 \%$, the critical point is absent to $80 \%$ of the HMW-HA content (line 1).

The relationships between the relative viscosity and concentration of LMW-HA solutions, along with their mixtures, are shown in Figure 3. The critical concentration manifests for LMW-HA mixtures with a content of HMW-HA of more than $40 \%$. When a solution contains $60 \%$ of HMW-HA, the critical concentration appears in the region of $0.05 \%$ (line 4 ), and at $80 \%$ of acid - at $0.0156 \%$ (line 5). The presence of critical values indicates that the content of HMW-HA with high molecular mass becomes sufficient for the appearance of conformational difficulties in the implementation of intermolecular interactions between molecular chains in solution.

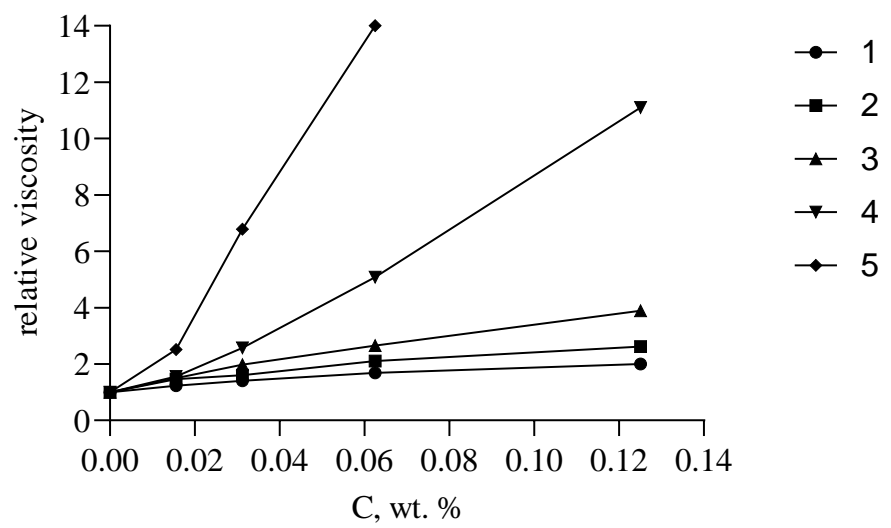

Figure 3. Relationship between the relative viscosity $\left(\eta_{r e l}\right)$ and solution concentrations for LMW-HA (1) and its mixtures with HMW-HA at a ratio of HMW-HA/LMW-HA 20/80 (2), 40/60 (3), 60/40 (4) and 80/20 (5) on the concentration of solutions. 

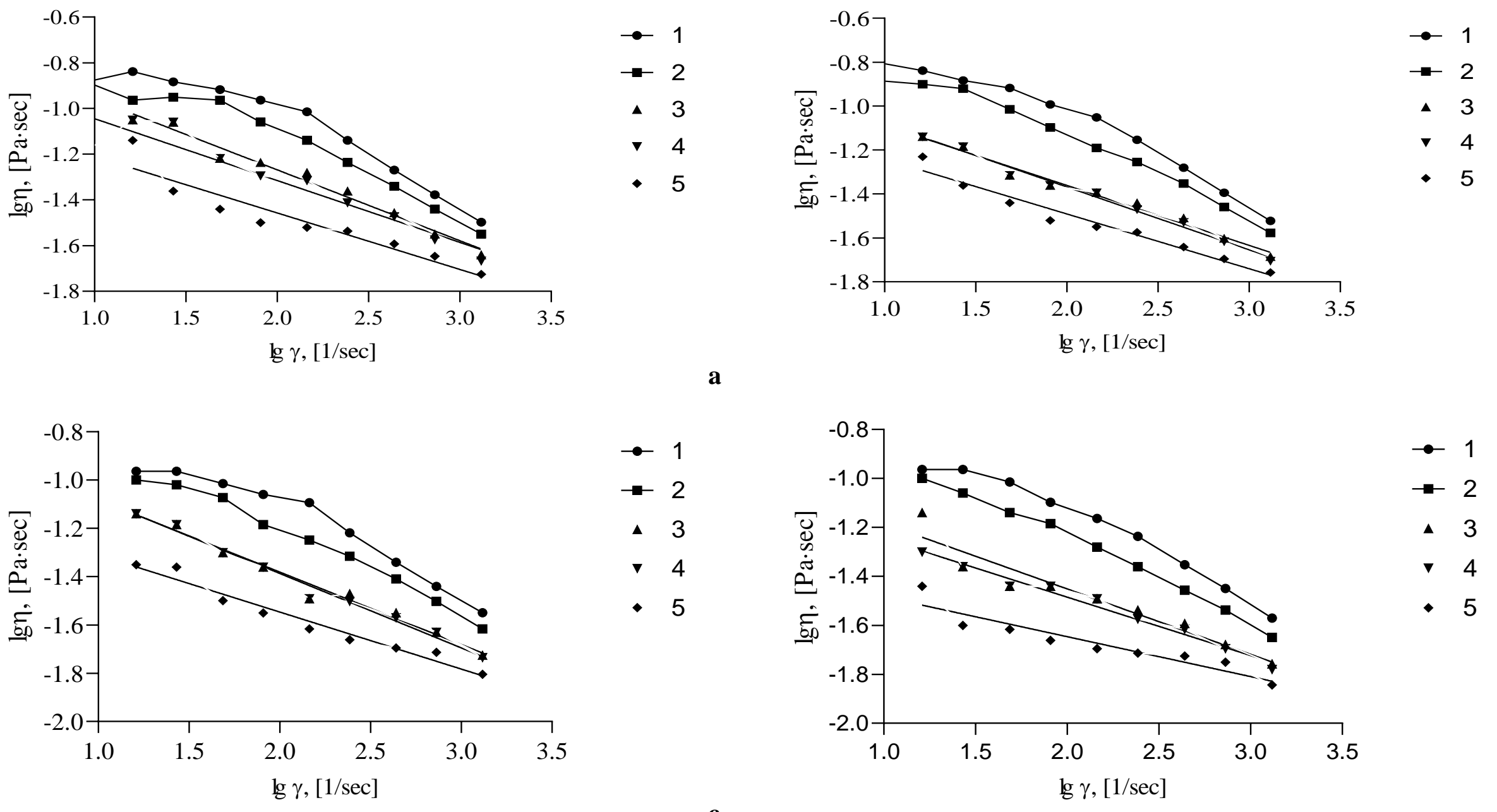

Figure 4. Relationship between viscosity change $(\eta)$ and the shear rate for $0.125 \%$ HMW-HA solutions (1) and its mixtures with LMW-HA at a ratio of HMW-HA/LMW-HA: 2 $80 / 20 ; 3-70 / 30 ; 4-60 / 40 ; 5-50 / 50$. Temperature: $\mathrm{a}-20^{\circ} \mathrm{C} ; \mathrm{b}-25^{\circ} \mathrm{C} ; \mathrm{c}-30{ }^{\circ} \mathrm{C} ; \mathrm{d}-35^{\circ} \mathrm{C}$. 
The forces of intermolecular interaction in dilute polymer solutions are very weak, therefore, the formation of sufficiently strong fluctuation networks is not observed. Figure 3 illustrates the relationship between the viscosity of HMW-HA along with its LMW-HA mixtures and shear rate within the ratio of HMW/LMW acids 80/20 $\rightarrow 50 / 50$ at a temperature of $20-35{ }^{\circ} \mathrm{C}$. Diluted HA solutions exhibit the properties of pseudoplastic fluids with a chaotic arrangement of macromolecules, which are similar to Newtonian fluids at low shear rates. An increase in the shear rate leads to the orientation of macromolecules in the flow direction resulting in viscosity reduction of solutions. In turn, an increase of LMW-HA content in solution leads to weakening the already weak intermolecular bonds (lines 2-5), making the flow closer to the Newtonian one. Notably, at 30 and $40 \%$ of LMW-HA content in the mixtures, the given relationships (lines 3 and 4) practically coincide that correspond to the HMW-HA critical content region. At a content of LMW-HA of more than $50 \%$, the solutions flow almost like Newtonian liquids.

Mathematical relationships 1-5 (Figure 4) with the approximation coefficients satisfactorily fit on a line in the coordinates $\lg \eta-\lg \dot{\gamma}$ described by the equation of the seconddegree polynomial $\mathrm{y}=a \mathrm{x}^{2}+b \mathrm{x}+c$, where $\mathrm{y}=\lg \eta, \mathrm{x}=\lg \dot{\gamma}$. The calculated values of the coefficients $a, b$ and constant $c$ are given in Table 1 .

Table 1. Values of coefficients in the equation $\mathrm{y}=a \mathrm{x}^{2}+b \mathrm{x}+c$, where $\mathrm{y}=\lg \eta[\mathrm{Pa} \cdot \mathrm{s}] ; \mathrm{x}=\lg \gamma\left[\mathrm{s}^{-1}\right]$.

\begin{tabular}{|c|c|c|c|c|c|}
\hline \multirow[t]{2}{*}{ Ratio, \% } & \multirow{2}{*}{$\begin{array}{c}\text { Coefficient values } \\
\text { and } \mathbf{R}^{2^{*}}\end{array}$} & \multicolumn{4}{|c|}{ Temperature, ${ }^{\circ} \mathrm{C}$} \\
\hline & & 20 & 25 & 30 & 35 \\
\hline \multirow[t]{4}{*}{$100 / 0$} & $a$ & -0.1443 & -0.0801 & -0.1445 & -0.1105 \\
\hline & $b$ & 0.2738 & -0.0231 & 0.3032 & 0.1481 \\
\hline & $c$ & -0.9647 & -0.6606 & -1.1097 & -0.9649 \\
\hline & $\mathrm{R}^{2}$ & 0.9945 & 0.9875 & 0.9926 & 0.9972 \\
\hline \multirow[t]{4}{*}{$80 / 20$} & $a$ & -0.1273 & -0.0594 & -0.0661 & -0.0495 \\
\hline & $b$ & 0.2180 & -0.0955 & -0.0439 & -0.1246 \\
\hline & $c$ & -1.0122 & -0.6961 & -0.8357 & -0.7789 \\
\hline & $\mathrm{R}^{2}$ & 0.9900 & 0.9907 & 0.9954 & 0.9993 \\
\hline \multirow[t]{4}{*}{$70 / 30$} & $a$ & -0.0438 & -0.0075 & 0.0179 & 0.0440 \\
\hline & $b$ & -0.1208 & -0.2405 & -0.3773 & -0.4571 \\
\hline & $c$ & -0.8361 & -0.8472 & -0.7044 & -0.7286 \\
\hline & $\mathrm{R}^{2}$ & 0.9838 & 0.9825 & 0.9816 & 0.9290 \\
\hline \multirow[t]{4}{*}{$60 / 40$} & $a$ & -0.0101 & -0.0069 & 0.0179 & -0.0386 \\
\hline & $b$ & -0.2810 & -0.2562 & -0.3773 & -0.0715 \\
\hline & $c$ & -0.6841 & -0.8254 & -0.7044 & -1.1736 \\
\hline & $\mathrm{R}^{2}$ & 0.9843 & 0.9880 & 0.9816 & 0.9875 \\
\hline \multirow[t]{4}{*}{$50 / 50$} & $a$ & 0.1667 & -0.0614 & 0.0523 & 0.0487 \\
\hline & $b$ & -0.9296 & -0.5143 & -0.4613 & -0.3731 \\
\hline & $c$ & -0.3147 & -0.7296 & -0.8511 & -1.1121 \\
\hline & $\mathrm{R}^{2}$ & 0.9312 & 0.9787 & 0.9778 & 0.9045 \\
\hline
\end{tabular}

$* \mathrm{R}^{2}$ - approximation confidence value.

The linear relationships $\lg \eta-\lg$ constructed from the calculated data are shown in Figure 5. Temperature change within $20-35{ }^{\circ} \mathrm{C}$ insignificantly affects the nature of solutions' rheological behavior. 


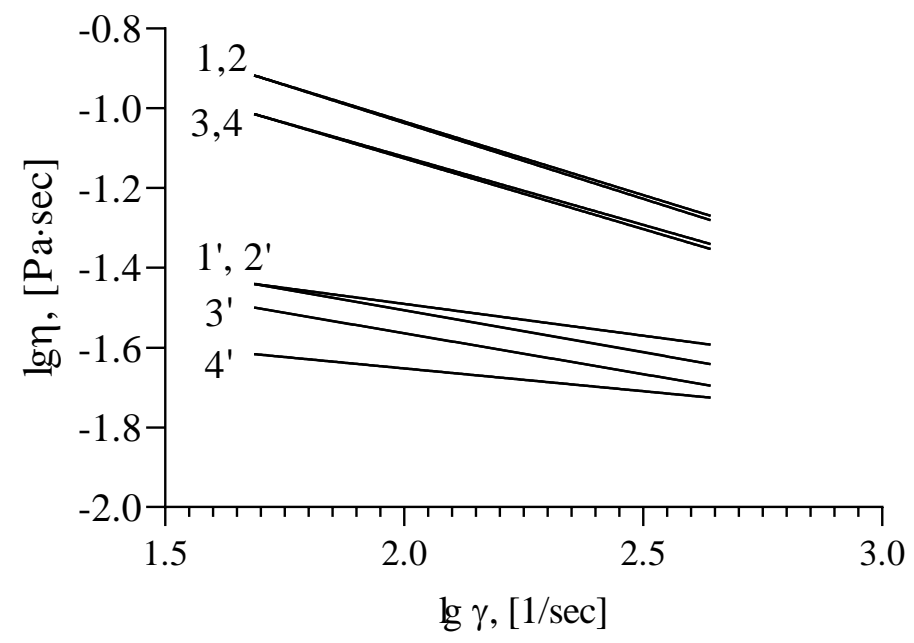

Figure 5. Relationship between $\lg \eta$ and $\lg \dot{\gamma}$ for $0.125 \%$ HMW-HA solutions (1-4) and its mixtures with LMW-HA at a ratio of 50/50 (1'-4'). Temperature: $1,1^{\prime}-20^{\circ} \mathrm{C} ; 2,2^{\prime}-25^{\circ} \mathrm{C} ; 3,3^{\prime}-30{ }^{\circ} \mathrm{C} ; 4,4^{\prime}-35^{\circ} \mathrm{C}$.

The relationship between $\log \eta$ and $\log$ for $0.125 \%$ solutions of HMW-HA (lines 1 and 2) with LMW-HA at a ratio of 50/50 (lines $1^{\prime}$ and $2^{\prime}$ ) at 20 and $25{ }^{\circ} \mathrm{C}$ almost coincide. A decrease in the viscosity of HMW-HA solution (lines 3 and 4) was observed within the temperature rise from 25 to $30{ }^{\circ} \mathrm{C}$ without changing the nature of the rheological behavior (tangent of the slope for lines 1, 2 and 3, 4 is essentially the same). An increase in temperature from 30 to $35^{\circ} \mathrm{C}$ (lines 3 ' and $4^{\prime}$ ) has a slightly greater effect on the rheological behavior nature of HMW-HA and LMW-HA mixture at their ratio 50/50.

Based on the rheology of polymer systems, the relationships between effective viscosity $\left(\eta_{\text {eff }}\right)$ and the shear rate $(\dot{\gamma})$ can be described by Ostwald-de Waele equation $\eta_{\text {eff }}=K \cdot \gamma^{\mathrm{n}-1}$. The values of coefficient $\mathrm{K}$ and flow index $\mathrm{n}$ calculated according to the experimental data for the HMW-HA solutions and its mixtures with LMW-HA at $20^{\circ} \mathrm{C}$ are given in Table 2.

Table 2. Values of coefficient $\mathrm{K}$ and flow index $\mathrm{n}$ for the HMW-HA solutions and mixtures with LMW-HA at $20^{\circ} \mathrm{C}$.

\begin{tabular}{c|c|c|c|c|c}
\multirow{2}{*}{ Coefficient } & \multicolumn{5}{|c}{ Ratio of HMW-HA/LMW-HA in 0.125\% solutions } \\
\cline { 2 - 6 } & $100 / 0$ & $80 / 20$ & $70 / 30$ & $60 / 40$ & $50 / 50$ \\
\hline $\mathrm{K}$ & 0.67 & 0.65 & 0.62 & 0.61 & 0.59 \\
\hline $\mathrm{n}$ & 0.53 & 0.71 & 0.76 & 0.77 & 0.78
\end{tabular}

\section{Conclusions}

The current study analyzed the rheological properties of high (HMW-HA) and low molecular weight (LMW-HA) hyaluronic acid solutions and their mixture in different concentrations. Based on matrix-assisted laser desorption ionization mass spectrometry (MALDI-MS) the molecular weight and polydispersity of LMW-HA have been established. The main findings are summarized as follows: diluted solutions of LMW-HA $\left(\overline{M_{n}}=10^{3}\right)$ with a concentration of $0.0156-0.125 \%$ are semi-structured liquids with a slight viscosity anomaly; HMW-HA $\left(\overline{M_{n}}=10^{6}\right)$ introduction into LMW-HA solutions at a certain (critical) content leads to a sharp increase in viscosity; the value of the critical concentration for ratio 60/40 (HMW$\mathrm{HA} / \mathrm{LMW}-\mathrm{HA}$ ) is at the level of $0.05 \%$, and for ratio $80 / 20-0.0156 \%$; rheological measurements demonstrated the pseudoplastic nature of the flow for HMW-HA solutions and its mixtures with LMW-HA; the abovementioned solutions possess identical rheological behavior within the temperature range $20-35^{\circ} \mathrm{C}$; the possibility of using Ostwald-de Waele 
equation $\eta=K \cdot \gamma^{\mathrm{n}-1}$ for the mathematical description of relationships between the effective viscosity of hyaluronic acid solutions and the shear rate has been revealed.

\section{Funding}

This research received no external funding.

\section{Acknowledgments}

This research has no acknowledgments.

\section{Conflicts of Interest}

The authors declare no conflict of interest.

\section{References}

1. Lequeux, I.; Ducasse, E.; Jouenne, T.; Thebault, P. Addition of antimicrobial properties to hyaluronic acid by grafting of antimicrobial peptide. European Polymer Journal 2014, 51, 182-190, https://doi.org/10.1016/j.eurpolymj.2013.11.012.

2. Lin, W.; Liu, Z.; Kampf, N.; Klein, J. The Role of Hyaluronic Acid in Cartilage Boundary Lubrication. Cells 2020, 9, 1606, https://doi.org/10.3390/cells9071606.

3. Heymann, D.; Vidal, L.; Or, Y.; Shoham, Z. Hyaluronic acid in embryo transfer media for assisted reproductive technologies. The Cochrane database of systematic reviews 2020, 9, CD007421, https://doi.org/10.1002/14651858.CD007421.pub4.

4. Gupta, R.C.; Lall, R.; Srivastava, A.; Sinha, A. Hyaluronic acid: molecular mechanisms and therapeutic trajectory. Frontiers in veterinary science 2019, 6, 192, https://doi.org/10.3389/fvets.2019.00192.

5. Cui, Y.; Wang, F.; Voorhees, J.J.; Fisher, G.J. Rejuvenation of aged human skin by injection of cross-linked hyaluronic acid. Plastic and reconstructive surgery, 2021, 147, 43S-49S, https://doi.org/10.1097/PRS.0000000000007620.

6. Papakonstantinou, E.; Roth, M.; Karakiulakis, G. Hyaluronic acid: A key molecule in skin aging. Dermatoendocrinology 2012, 4, 253-258, https://doi.org/10.4161/derm.21923.

7. Larrañeta, E.; Henry, M.; Irwin, N.J.; Trotter. J.; Perminova, A.A.; Donnelly, R.F. Synthesis and characterization of hyaluronic acid hydrogels cross-linked using a solvent-free process for potential biomedical applications. Carbohydrate polymers 2018, 181, 1194-1205, https://doi.org/10.1016/j.carbpol.2017.12.015.

8. Short, A.R.; Koralla, D.; Deshmukh, A.; Wissel, B.; Stocker, B.; Calhoun, M.; Dean, D.; Winter, J.O. Hydrogels that allow and facilitate bone repair, remodeling, and regeneration. Journal of materials chemistry B 2015, 3, 7818-7830, https://doi.org/10.1039/C5TB01043H.

9. Dovedytis, M.; Liu, Z.J.; Bartlett, S. Hyaluronic acid and its biomedical applications: A review. Engineered Regeneration 2020, 1, 102-113, https://doi.org/10.1016/j.engreg.2020.10.001.

10. Huynh, A.; Priefer, R. Hyaluronic acid applications in ophthalmology, rheumatology, and dermatology. Carbohydrate research 2020, 489, 107950, https://doi.org/10.1016/j.carres.2020.107950.

11. Salwowska, N.M.; Bebenek, K.A.; Żądło, D.A.; Wcisło-Dziadecka, D.L. Physiochemical properties and application of hyaluronic acid: a systematic review. Journal of cosmetic dermatology 2016, 15, 520-526, https://doi.org/10.1111/jocd.12237.

12. Kojima, T.; Nagata, T.; Kudo, H.; Müller-Lierheim, W.; van Setten, G. B.; Dogru, M.; Tsubota, K. The effects of high molecular weight hyaluronic acid eye drop application in environmental dry eye stress model mice. International journal of molecular sciences 2020, 21, 3516, https://doi.org/10.3390/ijms21103516.

13. Williams, A.C.; Barry, B.W. Penetration enhancers. Advanced drug delivery reviews 2004, 56, 603-618, https://doi.org/10.1016/j.addr.2003.10.025.

14. Witting, M.; Boreham, A.; Brodwolf, R.; Vávrová, K.; Alexiev, U.; Friess, W.; Hedtrich, S. Interactions of hyaluronic acid with the skin and implications for the dermal delivery of biomacromolecules. Molecular pharmaceutics 2015, 12, 1391-401, https://doi.org/10.1021/mp500676e. 
15. Nesterkina, M.; Kravchenko, I. Analgesic activity of novel GABA esters after transdermal delivery. Natural product communications 2016, 11, 1419-1420, https://doi.org/10.1177/1934578X1601101001.

16. Nesterkina, M.; Kravchenko, I. Synthesis and pharmacological properties of novel esters based on monocyclic terpenes and GABA. Pharmaceuticals 2016, 9, 32, https://doi.org/10.3390/ph9020032.

17. Snetkov, P.; Zakharova, K.; Morozkina, S.; Olekhnovich, R.; Uspenskaya, M. Hyaluronic acid: the influence of molecular weight on structural, physical, physico-chemical, and degradable properties of biopolymer. Polymers 2020, 12, 1800, https://doi.org/10.3390/polym12081800.

18. Dicker, K.T.; Gurski, L.A.; Pradhan-Bhatt, S.; Witt, R.L.; Farach-Carson, M.C.; Jia, X. Hyaluronan: a simple polysaccharide with diverse biological functions.Acta biomaterialia 2014, 10, 1558-1570, https://doi.org/10.1016/j.actbio.2013.12.019.

19. Fallacara, A.; Baldini, E.; Manfredini, S.; Vertuani, S. Hyaluronic acid in the third millennium. Polymers (Basel) 2018, 10, 701, https://doi.org/10.3390/polym10070701.

20. Joyce, K.; Fabra, G.T.; Bozkurt, Y.; Pandit, A. Bioactive potential of natural biomaterials: identification, retention and assessment of biological properties. Signal Transduction and Targeted Therapy 2021, 6, 122, https://doi.org/10.1038/s41392-021-00512-8.

21. Makvandi, P.; Caccavale, C.; Della Sala, F.; Zeppetelli, S.; Veneziano, R.; Borzacchiello, A. Natural formulations provide antioxidant complement to hyaluronic acid-based topical applications used in wound healing. Polymers 2020, 12, 1847, https://doi.org/10.3390/polym12081847.

22. Kim, D.J.; Jung, M.Y.; Pak, H.J.; Kim, M.; Chuck, R.S.; Park, C.Y. Development of a novel hyaluronic acid membrane for the treatment of ocular surface diseases. Scientific Reports 2021, 11, 2351, https://doi.org/10.1038/s41598-021-81983-1.

23. Hodovana, O.I.; Klyuchivska, O.Yu.; Godovanyi, O.V.; Miahkota, O.S.; Mitina, N.Ye.;.Konstantinova, T.Ye.; Kotsyumbas, H.I.; Zaichenko, A.S.; Stoika, R.S. Development of novel $\mathrm{ZrO}_{2}-\mathrm{Gd}_{2} \mathrm{O}_{3}$ nanocomposite functionalized with hyaluronic acid and its application for repair of bone defects in experimental animals. Letters in Applied NanoBioScience 2016, 5, 397-405, https://doi.org/10.33263/LIANBS53.397405. 